\title{
Kosmo-politische Ereignisse. Zur sozialen Topologie von SARS
}

\author{
Von Michael Schillmeier und Wiebke Pohler
}

\begin{abstract}
Zusammenfassung: Die gesellschaftliche Brisanz von SARS (Severe Acute Respiratory Syndrom) zeigte sich zunächst an dem fehlenden Wissen über Ursache, Diagnosemöglichkeit und Evolution dieser hoch ansteckenden und lebensbedrohenden Erkrankung. SARS wurde als globale Gefahr wahrgenommen, da das Virus sich entlang internationaler Flugrouten auszubreiten vermochte und eine SARS Pandemie nicht auszuschließen war. Die grenzüberschreitende Migration von SARS wurde dadurch zu einem kosmo-politischen Ereignis, das individuelles Leben gefährdete und dem es gelang, tradierte gesellschaftliche Ordnungsmechanismen und -kalküle zu verunsichern und zu verändern. Anknüpfend an den >spatial turn< innerhalb der sozialwissenschaftlichen Forschung versuchen wir die sozial-räumliche Bedeutung (Topologie) von SARS zu rekonstruieren. Das Phänomen SARS lässt sich gesellschaftswissenschaftlich nur ungenügend fassen, würde man methodologisch die soziale Dimension von SARS im Unterschied zur stofflichmaterialen und individuell-körperlichen Dimension konzipieren. Die Migration von SARS zeigt, so unsere These, wie der Objektbereich sozialwissenschaftlicher Methoden in Frage gestellt wird, da er in der Verknüpfung mit dem Nicht-Sozialen als erklärungsbedürftig erscheint. Unser methodologischer Vorschlag ist, die topologische Komplexität von SARS als Akteur-Netzwerk zu erfassen. Ein Akteur-Netzwerk reflektiert ein prozessuales Raumkonzept, das es erlaubt, gesellschaftswirksame Akteure als die Verknüpfung von menschlichen und nicht-menschlichen Relationen sichtbar zu machen.
\end{abstract}

\section{Global Assemblages}

Am Ende des Jahres 2002 traten in der südchinesischen Provinz Guangdong die ersten Fälle einer neuartigen Atemwegserkrankung auf, die den Namen SARS (Severe Acute Respiratory Syndrom) erhalten sollte. Die Neuartigkeit von SARS machte sich an den unbekannten Ursachen für diese hoch ansteckende und lebensbedrohende Erkrankung fest. Ihre besondere Gefährlichkeit lag darin, dass sie sich entlang internationaler Flugrouten auszubreiten vermochte und die Möglichkeit einer SARS Pandemie nicht auszuschließen war. Am 15. März 2003 landete in Frankfurt am Main ein Flugzeug aus New York. An Bord befanden sich drei SARS-Verdachtspatienten. Es handelte sich dabei um einen Arzt aus Singapur, der in New York an einem Kongress teilgenommen hatte und der zusammen mit seiner Frau und seiner Schwiegermutter reiste. Der Arzt hatte zuvor in Singapur Patienten mit einer unbekannten Atemwegserkrankung behandelt. In New York erkrankte er und suchte einen Arzt auf. Dieser stellte die Diagnose einer Lungenentzündung und behandelte diese, doch die Therapie schlug nicht an. Daraufhin beschloss der Arzt aus Singapur die Heimreise anzutreten, um sich daheim auszukurieren. Vor seinem Abflug informierte er jedoch seine Heimatklinik über seine Erkrankung. In der Zwischenzeit häuften sich bei den lokalen Büros der Weltgesundheitsorganisation (WHO) die Meldungen über zahlreiche, ungewöhnlich verlaufende Atemwegserkrankungen in chinesischen Krankenhäusern. Die WHO rief daraufhin am 13. Februar 2003 einen weltweiten Alarm aus (WHO 2003a).

Durch diesen Alarm aufmerksam geworden, informierte auch die Singapurer Klinik die WHO über die Erkrankung eines ihrer Mitarbeiter. Die WHO gab diese Information an ihre lokalen Partner in New York weiter, die nun versuchen, den Arzt und seine Familie ausfindig zu machen. Die Befürchtungen gehen dahin, dass bei weiteren Infektionen durch den Arzt auf dem Flug von New York die Erkrankung in die ganze Welt verschleppt werden könnte. Der Flughafen Frankfurt am Main ist eine Zwischenstation des Fluges von New York. Hier steigen die Passagiere um und reisen dann in die verschiedensten Länder weiter.

Soziale Welt 57 (2006), S. $331-349$ 
Nachdem die Maschine, in der sich der Arzt mit seiner Familie befand, in New York bereits gestartet war, wurde das Stadtgesundheitsamt in Frankfurt am Main über dieses Flugzeug mit den Verdachtspatienten informiert, um beim Zwischenstopp der Maschine in Frankfurt reagieren zu können. Das Flugzeug wurde bei seiner Landung am Frankfurter Flughafen sofort unter Quarantäne genommen. Die Verdachtspatienten wurden an der Frankfurter Universitätsklinik aufgenommen und behandelt. Auch die anderen Passagiere des Fluges mussten sich vor ihrer Weiterreise einem Gesundheitscheck unterziehen.

Die schnelle Identifizierung von SARS-Patienten bzw. SARS-Verdachtsfällen und eine entsprechende Isolierung dieser Personen war in Bezug auf das Risiko der Ausbreitung von SARS deshalb so entscheidend, da durch die Möglichkeit der Krankheitsübertragung auf andere immer ein Risiko für die welt-öffentliche Gesundheit bestand. Jeder neue Fall von SARS erhöhte somit das Risiko einer globalen Ausbreitung. Dass es dennoch zu einer erfolgreichen Eindämmung der Migration von SARS kam, ist insbesondere auf die unterschiedlichen, aber hochwirksamen Kontroll-, Überwachungs- und Isolationstechnologien zurückzuführen sowie auf die Bildung transnationaler, virtueller medizinwissenschaftlicher Netzwerke, deren Stärke insbesondere darin lag, auf aktuelle Problemlagen schnell - auf der Basis von day-to-day Entscheidungen - reagieren zu können. Zwischen dem ersten Fall von SARS in der südchinesischen Provinz Guangdong im November 2002 und dem von der WHO ausgesprochenen weltweiten Ende des Ausbruchs im Juli 2003 kam es zu mehr als 8000 registrierten Ansteckungen und ca. 800 Todesfällen. Im Vergleich dazu hatte die Grippewelle in Deutschland im Jahre 2004/05 ca. 15000 Todesopfer gefordert.

Wie immer man SARS einzuschätzen vermag, die gesellschaftliche Brisanz ist in dem globalen Bedrohungs- und Gefährdungspotential für menschliches (Zusammen-)Leben (Baehr 2005) und der dadurch ausgelösten transnationalen Konflikt- und Kommunikationsdynamik zu sehen. SARS hat nicht nur Menschenleben gekostet; der Migration von SARS ist es auch gelungen, zum einen lokale wie globale gesellschaftliche Praktiken zu verunsichern, zu verändern und neu zu konstituieren, und zum anderen die tradierten Beobachtungs-, Erklärungsund Interpretationsmuster gesellschaftlicher Zusammenhänge fragwürdig erscheinen zu lassen.

\section{Aiwa Ong beschreibt SARS als}

$\gg .$. global assemblage, or a mobilization of significant connections among diverse elements that have open-ended effects on the meaning of individual and social life. (...) the focus on assemblage reveals how actors - including social analysts - define and respond by assembling diverse resources in a contingent and provisional manner, with varying effects on emerging forms of modern ways of living.« (Ong 2004: 81).

Solche global assemblages wie SARS, deren Folgen den gesellschaftlichen Beobachter sowie den Beobachtungsgegenstand in Frage stellen, wollen wir kosmo-politische Ereignisse nennen. Kosmo-politische Ereignisse, so unsere These, sind grenzüberschreitende, öffentliche Akteure, die sowohl gesellschaftliche Ordnungsmuster als auch die Routinen ihrer Beschreibung kontingent erscheinen lassen und reformulieren. Die Bedeutung von SARS liegt nun darin, dass SARS sich paradoxerweise der Macht sozialer Zuschreibung >Sinn< zu kommunizieren entzieht. Kosmo-politische Ereignisse agieren vielmehr als paradoxe Sinnstifter, die soziale Relevanz in dem Moment aktualisieren, in welchem sie den Kosmos der jeweiligen Sinn- und Wissensarrangements sozialer Praxis verunsichern und gefährden. An so unterschiedlichen Ereignissen wie z.B. Tschernobyl, AIDS oder Stammzellenforschung lässt sich dies bereits deutlich zeigen (Jasanoff/Long Martello 2004; Ong/Collier 2005; Petryna 2002; Preda 2004). 


\section{Topologische Komplexität}

Die WHO beschreibt auf ihrer homepage die Besonderheit von SARS:

»It is clear that the responsibility for containing the emergence of any new infectious disease showing international spread lies with all countries. In a world where all national borders are porous when confronted by a microbial threat, it is in the interest of all populations for countries to share the information they may have as soon as it is available. In so doing, they will allow both near and distant countries - all neighbors in our globalized world - to benefit from the understanding they have gained.«(WHO 2003b: 9)

Wie will man nun die durch SARS initiierte Kosmo-politisierung gesellschaftlicher Wirklichkeit und die damit verknüpfte Infragestellung soziologischer Beobachtungsroutinen methodisch in den Griff bekommen? Eine attraktive Möglichkeit dies zu tun besteht darin, die Topologie von SARS zu beschreiben. Wenn wir von Topologie sprechen, schließen wir uns dem >spatial turn< innerhalb der sozialwissenschaftlichen Forschung an. Dieser versucht die Grenzen der methodischen wie theoretischen Anstrengungen, gesellschaftliche Zusammenhänge anhand regionaler und territorialer Metaphern und Konzepte zu beschreiben, kritisch zu reflektieren (Barabasi 2002; Czarniawska/Sevón 2005; Hetherington/Lee 2000; Law 2004; Law/Mol 2001; Callon/Law 2004; Moreira 2004a; 2004b; Schillmeier im Erscheinen; Thrift 1996; Urry 2000; 2005).

Interessant an einer topologischen Methodologie ist zunächst, dass diese die Herstellung von sozialen Räumen zu erfassen versucht, ohne zu deren Beschreibung allein auf geometrische Metaphern und Methoden, d.h. auf Entfernungen, Maße oder Größen, angewiesen zu sein. Vielmehr liegt der Fokus auf der Fabrikation einer Vielfalt von sich überlagernden Räumen, die »nonisomorphic with standard units of analysis« (Collier/Ong 2005: 3) sind. Eine topologische Methodologie widmet sich den je spezifischen räumlichen Merkmalen, die den beobachteten Objekten ihre gesellschaftliche Präsenz sichern (Law 1999). Euklidisch, territorial und regional bestimmte Räume benennen dabei nur spezifische topologische Formen, um gesellschaftliche Zusammenhänge eingrenzbar und beschreibbar, messbar und vergleichbar zu machen. Eine Alternative dazu bietet der Begriff des Akteur-Netzwerkes (Callon 1991; Latour 1996; 2001; 2005), der versucht ein prozessuales Raumkonzept sichtbar zu machen. Akteur-Netzwerke verweisen auf Elemente, die ihre Stabilität und >räumliche Integrität< durch ihre Position in einem Set von Verbindungen und Beziehungen gewinnen (Law 1999: 6). ${ }^{1}$ Territorial gedachte und konzipierte soziale Räume erscheinen so als ein Effekt von einer Vielzahl von miteinander verbundenen Relationen und generieren auf diese Weise räumliche Stabilität und Integrität. Solche »layered spaces« (Moreira 2004b) machen die topologische Komplexität gesellschaftlicher Praxis aus und entziehen sich dadurch immer auch einer vereinheitlichenden Perspektive (Taylor 2001; Urry 2003).

Dies wollen wir kurz an dem Verhältnis von Lokalität und Globalität illustrieren. Zentral für eine Methodologie topologischer Dynamiken ist, dass die Spielarten global gedachter Räume - sei es in der Form >Kontext<, >Subjekt<, >Objekt<, >Mensch<, >Nation<, >Welt<, >Sozialität<, >Natur<, >Kosmos< - immer nur als Folge des Lokalen, d.h. als selektives Artikulieren lokaler Komplexität virulent werden. Topologisch gedacht kann das Globale oder Globalität demzufolge als standardisierte und formalisierte Übersetzung lokaler Komplexität verstanden werden (Latour 2001). Globalisierung, also weltweites Handeln auf Distanz, ist hierfür ein gutes Beispiel. Paradoxerweise zeigt sich die topologische Komplexität von Globalisierung gerade im Verschwinden des einen globalen Raumes. Letzterer artikuliert sich

1) Zur kritischen Diskussion des Begriffs >Akteur-Netzwerk< vgl. Latour (2005), Law/Hassard (1999) und Lee/Brown (1994). 
vielmehr als hyperkomplexe Vermittlung von weltweit lokal vernetzten Kommunikationsund Transportstrukturen, die dadurch lokal globale Mobilität und Kommunikation ermöglichen. Man spricht in diesem Zusammenhang dann auch von >Weltgesellschaft $<$, in der es keine eine Welt mehr gibt, in der es stattdessen zu einer Gleichzeitigkeit multipler >Gegenwarten< dieser Weltgesellschaft kommt (Nassehi 2003: 188ff).

Man kann nun aus topologischer Sicht sagen: Im Zuge der Globalisierung dis/lokalisiert sich das Globale. Dis/lokalisierung meint, dass sich das Globale nicht mehr im Unterschied zum Lokalen verorten lässt, so, als ob man zwei territorial abgeschlossene Regionen miteinander vergleicht. Das Globale kann überhaupt keinem >natürlichen< Ort mehr zugewiesen werden. Vielmehr lässt sich das Besondere des Globalen nur mehr als Folge von Lokalisierung denken. Es kommt so zu einer Überlappung und Multiplikation lokaler/globaler Verknüpfungen. Topologisch gesehen, kann auch >das Lokale< dann nur noch als Vermittlung und Verknüpfung solcher heterogenen Dis/lokalisierungen gedacht werden. Somit lässt sich die Differenz zwischen dem >Globalen< und dem >Lokalen< nicht mehr als geometrisch gedachte Gegenüberstellung, als Entgegensetzung oder in Opposition zueinander konzipieren.

Auf die Frage hin, was dies für die topologische Komplexität des sozialen Raumes bedeutet, vermuten wir, dass >das Soziale< ebenfalls dem Prozess der Dis/lokalisierung des Globalen unterworfen ist. Durch Globalisierung wird eindringlicher denn je sichtbar, dass die Globalität des sozialen Raumes ebenfalls in Frage steht. Wir hatten betont, dass topologische Komplexität auf die Vermittlung und Verknüpfung von nicht-konformen Räumen verweist. Dies reflektiert das klassische Vorgehen der sozialwissenschaftlich begründeten Methoden, indem es dem sozialen Raum topologische Komplexität zuschreibt und dadurch den sozialen Raum - clare et distincte - von nicht-sozialen Dingen unterscheidet. Dem sozialen Raum wird ein eigener, abgetrennter Bereich zugestanden, der methodologisch seine räumlichen Grenzen mit dem Hinweis auf die Kontingenz der Welt sichert und sich so von Annahmen einer außer-sozialen, also natürlichen Weltordnung schützt und abgrenzt. Dadurch ist Welt entweder sozial oder natürlich und dies immer nur innerhalb der Grenzen der global gedachten Erklärungsmacht der Methodologie des sozialen Raumes.

Topologische Komplexität wird somit methodologisch durch die Unterscheidung vom Nicht-Sozialen gewonnen. Interessanterweise wird dadurch der sozialwissenschaftlichen Beobachtung ein >natürlicher< Objektbereich zugewiesen, der sich durch das Interesse am Nicht-Natürlichen ausweist. Unser methodologischer Vorschlag ist nun, topologische Komplexität auf den >natürlichen< Objektbereich des sozialen Raumes selbst anzuwenden. Methodisch bedeutet das, den Objektbereich der gesellschaftswissenschaftlichen Beobachtung nicht im Unterschied (Differenz als Opposition) zum >Nicht-Sozialen<, sondern in der Verknüpfung mit diesem zu denken und damit dessen Virulenz (van Loon 2002) sichtbar zu machen. Dadurch wird methodologisch der quasi-natürliche Objektbereich des sozialen Raumes in Frage gestellt und in der Verknüpfung mit dem Nicht-Sozialen erklärungsbedürftig.

Der Ausbruch von SARS hat diesen virulenten Charakter gerade im Gesundheitswesen betroffener Länder wie z.B. Kanada deutlich erkennen lassen. Dazu schreibt der kanadische ${ }^{2}$ >SARS Commission First Interim Report<:

»SARS demonstrated that hospitals and other health care facilities are not isolated institutions operating on their own. (...) Because SARS was such a difficult disease to diagnose, because there were no reliable lab tests, and because knowledge about the disease was rapidly evolving on a daily basis, there were disagreements from time to time between the reporting institution and public health officials as to whether a particular case was a case of

2) Kanada war neben China das am stärksten betroffene Land mit über 43 Todesfällen (Preiser 2004). 
SARS.«(Campbell, 2004: 147f). »(...) Viruses do not respect boundaries between municipal health units. The chain of provincial protection against the spread of infectious disease is only as strong as the weakest link in the 37 local public health units. A failure in one public health unit can spill into other public health units and impact the entire province and ultimately the entire country and the international community.«(Campbell, 2004: 201f).

Zusammenfassend kann man sagen: Topologische Räume sind ins Netzwerk gesetzte soziale und nicht-soziale Ereignisse, die paradoxerweise weder allgemeinen/globalen noch individuellen/lokalen Charakter besitzen. Damit wird gesellschaftliche Wirklichkeit nicht im Unterschied zur Natur, zum Psychischen oder zur sinnlichen Wahrnehmung konzeptualisiert, wie es der klassischen methodologischen Sicht einer >globalen Soziologie< entspricht (Turner 2006). Wie wir am Beispiel von SARS zeigen werden, ist die Methodologie des Akteur-Netzwerke(n)s eine Möglichkeit, die Quasi-Natürlichkeit des sozialen Raumes in Frage zu stellen.

Kosmo-politische Ereignisse wie SARS sind dafür symptomatisch, da sie, so unsere These, multiple und heterogene gesellschaftliche Ordnungstopologien entfalten, die sich nicht mehr durch einen »einfachen Ort« (Whitehead 1967) ${ }^{3}$ im Raum und in der Zeit methodisch kontrollieren lassen. Das meint: SARS lässt sich gesellschaftswissenschaftlich nur ungenügend fassen, würde man methodologisch die soziale Dimension von SARS im Unterschied zur stofflich-materialen Dimension oder im Unterschied zur individuell-körperlichen Dimension konzipieren und die soziale Dimension einseitig, quasi-natürlich zur Erklärung der beiden letzteren stilisieren. Dadurch wird ein weiteres Moment topologischer Komplexität sichtbar: Die gesellschaftliche Bedeutung von SARS lässt sich auch nicht im Unterschied zur oder in der Abstraktion von der zeitlichen Dimension denken. Kosmo-politische Ereignisse wie SARS stellen vielmehr das Ereignen selbst und die dadurch entstehende Multiplikation von gesellschaftlichen Räumen und Zeiten in den Mittelpunkt der Analyse. ${ }^{4}$ Der Begriff Akteur-Netzwerk scheint diesem eigentümlichen Ereignischarakter topologischer Komplexität zu entsprechen. Dabei ist zu beachten, dass weder der Begriff >Akteur $<$ noch der der >Netzwerke< der klassischen dialektischen Unterscheidung von Handlung und Struktur oder Individuum und Gesellschaft entspricht. Dazu Bruno Latour:

»Each locus can be seen as framing and summing up. »Actor« is not here to play the role of agency and »network « to play the role of society. Actor and network (...) designates two faces of the same phenomenon, like waves and particles, the slow realization that the social is a certain type of circulation that can travel endlessly without ever encountering either the micro-level - there is never an interaction that is not framed - or the macro-level - there are only local summing up which produce either local totalities (...) or total localities (...). (...) if there is no zoom going from the macro structure to micro interactions, if both micro and macro are local effects of hooking up to circulating entities, if contexts flow inside narrow conduits, it means that there is plenty of > space < in between the tiny trajectories of what could be called the local productions of »phusigenics«, »sociogenics« and »psychogenics«. (Latour 1999: 18-9).

3) Man benennt einen solchen >einfachen Ort< immer dann, sobald »one major characteristic which refers equally both to space and to time, and other minor characteristics are diverse as between space and time« (Whitehead 1967: 49). Die Beschreibung eines einfachen Ortes verweist auf die Tücken der »fallacy of misplaced concreteness « (1967: 51), wie Whitehead das nennt, sobald man das Konkrete durch das Abstrakte zu erklären versucht. Vgl. dazu auch Serres (2005).

4) Somit wird nicht nur die konventionelle Unterscheidung >sozial-zeitlich-sachlich< sozialkonstruktivistischer Provenienz unterlaufen, sondern damit verbindet sich auch ein gesteigertes Interesse an der Analyse der Multiplikation und Be- und Entschleunigung von Zeit(en). Vgl. dazu die Arbeiten von Paul Virilio aber auch die von Gleick (1999), Stengers (2004), Taylor (2001) und Urry (2002). 
Unter dem Einfluss von Akteur-Netzwerk-Ereignissen wie SARS lässt sich >das Soziale< nicht mehr als abstrakter globaler Raum (= einfacher Ort) im Unterschied zum und als Erklärung des Nicht-Sozialen (Sach- und Zeitdimension) heranführen, sondern wird selbst durch die Verknüpfung mit der Psycho-, Sach- und Zeitdimension erklärungsbedürftig. Gesellschaftliche Wirklichkeit wird dadurch zu einem dis/lokalisierten und heterogenen, d.h. zu einem zeit-räumlich und materialen multiplen Ereignis. Methodisch wichtig ist hierbei, dass dadurch sowohl der Beobachtungsgegenstand als auch der sozialwissenschaftliche Beobachter zu einem in Frage zu stellenden und in Frage gestellten gesellschaftlichen Akteur werden. Gerade Science, Technology \& Society (STS) hat zu dem »ontologischen Tanz (Cussins 1998) gesellschaftlicher Wirklichkeit bereits überzeugendes empirisches Material gesammelt (Callon 1998; Law 1991; 2002a; b; Mol 2002; Berg/Mol 1998; Law/Mol 2002; Strathern 1991; 1992).

Stellt man auf die Methodologie topologischer Räume um, so hat dies weitreichende Auswirkungen darauf, was man >Theorie < im Unterschied zur >Praxis $<$ nennt. Der soziologische Diskurs ist über weite Strecken von einem platonisch gedachten Theorieverständnis geprägt, das Theorie (theoria) als das kognitive Absehen (abstractio) und damit das Unterscheiden von sinnlichem, physiologischem Sehen, versteht. Der kontemplative Akt des Theoretisierens des Sozialen durch den soziologischen Beobachter unterscheidet soziale Praxis von sinnlicher, physiologischer Wahrnehmung und psychischen Vorgängen (Denken) als sozialen Prozess und umgrenzt und trennt dadurch - clare et distincte - den Bereich des Sozialen vom NichtSozialen. Gerade in dieser Abstraktionsfähigkeit von physiologischen und psychologischen Prozessen liegt der erkenntnistheoretische Gehalt, soziale >Dinge < nicht nur einfach zu benennen, sondern sie dadurch als Unterscheidung, als Differenz, sichtbar zu machen. ${ }^{5}$

Es ist die Stärke radikalen Soziologisierens - von Durkheim bis Luhmann -, einen klar bestimmten Begriff des Sozialen vorauszusetzen, der sich als autonome Sphäre (soziale Tatsache) oder aber als selbstreferentielle Kommunikation (Beobachten von Beobachtungen) von stofflich-materialen und psychischen Einheiten unterscheidet (Durkheim 1961; Luhmann 1992). Diese stabile und unverrückbare Einheit ist dabei nicht als Substanz zu denken, sondern verweist einerseits auf eine Unterscheidung als emergenten Prozess (Durkheim) und/ oder andererseits auf das operationale Unterscheiden (Form) je spezifischer Systemrationalitäten (Luhmann). So ein epistemologisches Verständnis von Sozialität erlaubt es dann, im Unterschied zu reinem Ontologisieren, Soziales als Soziales vorauszusetzen, d.h. vom Gleichen als Folge einer beobachtbaren und beobachteten Unterscheidung zu sprechen.

Demgegenüber konzentriert sich die hier vorgestellte topologische Methodologie auf die Zusammengehörigkeit von Theorie und Praxis als > Vernetzwerken < von Sozialem und NichtSozialem. Und das ist mehr, als das reine Theoretisieren und Soziologisieren sichtbar machen können. Während die klassische Soziologie also den Begriff des Sozialen zukunftsresistent an den Anfang und das Ende ihrer Beobachtungen über Gesellschaft (im Unterschied zur Natur) stellt, benennt unsere methodologische Argumentation das »Ende des Sozialen « (Latour 2000; 2001). Sprechen wir vom Ende des Sozialen, so wollen wir damit eine Methodologie benennen, der es nicht darum geht, gesellschaftliche Problemlagen in der Begrenzung, Identität oder Einheit der Differenz des Sozialen zu sehen. Vielmehr bestimmt sich gesellschaftliche Komplexität in der Nichtreduzierbarkeit auf rein soziale Phänomene.

Die damit verbundene topologische Komplexität wollen wir nun näher am Beispiel von SARS erläutern und mit dem Begriff >Akteur-Netzwerk< beschreiben.

5) Darüber sind sich viele spätestens seit Kant (1997) einig und verbinden damit wertvolle erkenntnistheoretische Einsichten über Modernität. Anstatt vieler vgl. Bateson/Bateson (1993), Luhmann (1992) und Nassehi (2003). 


\section{SARS-Akteur-Netzwerke}

SARS ist eine neuartige Erkrankung, die durch ein Virus ausgelöst wird. Viren sind dadurch gekennzeichnet, dass sie allein nicht lebensfähig sind. Sie verfügen zwar über eine eigene Erbinformation (DNA oder RNA), benötigen aber einen anderen lebenden Organismus (Zellen), um sich replizieren zu können und Nachkommenschaft zu bilden. Um sich also fortzupflanzen, bindet sich ein Virus mit Hilfe eines Rezeptors an eine Wirtszelle und nutzt dann den Zellabbau und -aufbau zur eigenen Vermehrung. Die Zelle nimmt in den Prozessen der Zellregeneration die Virusbestandteile auf und aus Zell- und Virusbausteinen entsteht ein neues Virus (= Virion) (Preiser/Rabenau/Doerr 2004). Um sich jedoch immer wieder mit neuen Zellen verbinden zu können, muss das Virus einen Weg der Übertragung finden. Bei SARS handelt es sich um eine Atemwegserkrankung. Das Virus wird dann in erster Linie über Atemwegssekrete (Rachentröpfchen) übertragen (Doerr 2003). Auf diese Weise wird es dem Virus möglich, sich von Körper zu Körper und quer zu den gesellschaftlich tradierten Grenzen auszubreiten.

Der Krankheitserreger von SARS wurde virologisch als SARS-Coronavirus charakterisiert, und es wird vermutet, dass das Virus sein Ursprungsreservoir bei Tieren hatte. Bei Laboruntersuchungen wurden bei verschiedenen Wildtieren, u.a. auch beim Larvenroller (»Zibetkatze«, Familie der Schleichkatzen), dem SARS-Coronavirus ähnliche Viren gefunden (Preiser 2004). In China werden eine Reihe von Wildtieren auf Märkten verkauft und gelten als Delikatesse. Epidemiologische Untersuchungen zu SARS ergaben, dass die frühen Fälle von SARS sehr häufig bei Personen auftraten, die direkten Kontakt zu wilden Tieren hatten. Dazu zählen insbesondere Tierhändler und Köche (Breiman u.a. 2003). Solange es bei den Tieren verblieb, war das Virus in Bezug auf den Menschen völlig folgenlos. Als jedoch das Virus die Artgrenze überschritt und mit dem menschlichen Organismus zusammenkam, entwickelte sich das Risiko der pandemischen Ausbreitung einer neuartigen und lebensbedrohlichen Krankheit. Die Übertragung des Erregers vom Tier zum Menschen wurde durch einen sehr engen Kontakt zwischen Mensch und Tier bewirkt.

Für den menschlichen Organismus stellt das Virus einen Fremdkörper dar, worauf das Immunsystem reagiert und als Abwehrreaktion schwerwiegende Erkrankungen verursachen kann. Das würde zunächst einmal nur bedeuten, dass das Virus den Menschen mehr oder weniger krank macht. Das Virus ist aber darauf angewiesen weitergegeben zu werden, um sich zu replizieren. Viren sind aus diesem Grund hochanpassungsfähig. Das SARS-Virus hat sich beim Übertreten vom Tier zum Menschen so verändert, dass es unabhängig von seinem Ursprungsreservoir existieren kann, indem es sich durch eine Weitergabe von Mensch zu Mensch repliziert. Die Gefahr einer pandemischen Ausbreitung von SARS ergab sich aus dieser Mutation des Virus und der damit verbundenen Möglichkeit der Weitergabe des Virus von Mensch zu Mensch in einer hochmobilen und globalisierten Welt - mit und ohne dessen explizite soziale Wahrnehmung.

Was zeichnet das Virus aus? Wir behaupten nun, dass es dessen Fähigkeit ist, Heterogenität, d.h. Ungleichartigkeit zu erkennen und für das eigene Sein zu übersetzen. Die Praxis des Virus, sich zu erhalten und auszubreiten, anerkennt dabei Uneinheitlichkeit und gebraucht die dadurch entstehenden Veränderungen (Virion) zur Stabilisierung des eigenen Seins und Tuns. Viren müssen das Andere (z.B. die Zelle) >begehren ‘, um das sein zu können, was sie sind. Darin ist ihre Virulenz zu sehen. Virulenz ist somit von der Praxis der Anerkennung des Anderen nicht zu trennen. Die prekäre Folge dieser Praxis besteht darin, dass das Virus dadurch aber immer auch den Kosmos, d.h. die Ordnung seiner selbst und die der anderen verändert. So verstanden, artikuliert sich durch die viröse Infektion menschlicher Körper die Macht von SARS als kosmo-politisches Ereignis und dem damit verbundenen Risiko ${ }^{6}$ einer 
epidemischen oder gar pandemischen Ausbreitung mit unvorhersehbaren Folgen für menschliches Leben und gesellschaftliche Organisation.

Dies ist jedoch nur möglich, wenn das Virus sich in Zirkulation, in Bewegung befindet und ganz bestimmte, hoch idiosynkratische Wege der Ausbreitung nutzen kann. Es braucht ein drittes Element, um das zu tun. ${ }^{7}$ Bei SARS erfolgt sowohl das Andocken im Körper selbst, als auch von Mensch zu Mensch durch bestimmte Körperbauteile (Rezeptoren) und Flüssigkeiten (Rachentröpfchen). Die Folgen der Zirkulation von SARS übersetzen sich dann in sozialen Kommunikationen über SARS, die wiederum andere Strategien und Technologien der Verbreitung von SARS (medizinisch, massenmedial, wissenschaftlich etc.) zur Folge haben. Damit können sich auch die Ziele der jeweiligen Strategien radikal ändern. War es zunächst die soziale Fähigkeit des Virus sich fortzupflanzen, ist es nun die soziotechnische Kommunikation über SARS dies zu verhindern.

Um das Virus zu identifizieren, wurden transnationale Netzwerke ins Leben gerufen, die eine sichere website benutzten:

»...to post electron microscopic pictures of candidate viruses, sequences of genetic material for virus identification and characterization, descriptions of experiments, and results. The well-guarded secret techniques that give each laboratory its competitive edge have been immediately and openly shared with others. Laboratories also quickly exchange various samples from patients and postmortem tissues. These arrangements have allowed the analysis of samples from the same patient simultaneously in several laboratories specialized in different approaches, with the results shared in real time. This collaboration has resulted in the identification of the suspected causative agent, and the development of three diagnostic tests, which unprecedented speed.«(WHO 2003b: 8)

Die Fähigkeit, durch Zirkulation Stabilität und Wandel zu initiieren, dabei einerseits heterogene Elemente zu verknüpfen, sichtbar zu machen als auch neu zu begründen und andererseits damit ganz bestimmte gesellschaftliche (menschlich und nicht-menschliche, soziale und nicht-soziale) Zusammenhänge und Bedingungen wichtig und/oder unwichtig, präsent und/ oder absent, sicher und/oder unsicher, lokal und/oder global erscheinen zu lassen, haben wir mit dem Begriff Akteur-Netzwerk beschrieben.

Verstanden als Akteur-Netzwerk ist die gesellschaftliche Bedeutsamkeit von SARS für Menschen nicht unabhängig von der Virulenz eines nicht-menschlichen Erregers zu denken (van Loon 2002). Die Möglichkeit einer weltweiten Ausbreitung war dabei in der Praxis der Vermehrung des Virus selbst, also in der Form lokaler, nicht-sozialer Infektionen angelegt. Die Zirkulation des Virus verband dann zunächst Tierkörper und menschliche Körper. Die

6) SARS war zu keiner Zeit ein kosmopolitischer Akteur im Sinne des medizinischen Diskurses. Dies würde eine tatsächliche globale Verbreitung voraussetzen. Die Besonderheit von SARS lag in dem Risiko, d.h. der Virtualität, sich global auszubreiten und hat gerade dadurch unsere gesellschaftlichen Routinen erschüttert und verändert.

7) Das wohl bekannteste gesellschaftswirksame Beispiel eines dritten Elements - >Geld - hat Georg Simmel bereits in seiner »Philosophie des Geldes« (Simmel 1989) entfaltet. Geld wird von Simmel als »wesenloses Wesen« (Simmel 1989: 691) bedeutsam, das als zirkulierendes Mittel die »Bedingtheit der Dinge « (Simmel 1989: 120) ausmacht. Als >absolute Zwischeninstanz<, als eben >dritte Instanz< erlaubt Geld soziale Praxis, welche mehr ist »als ein privater Vorgang zwischen zwei Individuen, der völlig in den individuellen Aktionen und Gegenreaktionen dieser beschlossen liegt « (Simmel 1989: 213). Ganz im Sinne eines Akteur-Netzwerkes lassen sich Subjekte, Objekte, Dinge dann nur »in Beziehungen und Prozesse« denken, als »eine auf- oder absteigende Reihe, in der jedes Glied von einem anderen abhängt und ein drittes von sich abhängen lässt: das mag inbezug auf räumliche Anordnung, auf kausale Energieübertragung, auf zeitliche Folge, auf logische Ableitung stattfinden« (Simmel 1989: 120, Hervorhebung im Original). 
Folge der Zoonose, also des Übergangs des Virus vom Tier zum Menschen, lag in der Evolution des Virus: Es entstand ein ontologisch neues Virus, das nun sowohl Bausteine des tierischen als auch des menschlichen Organismus enthielt. Die Veränderung des Virus wird durch die generell hochgradige Anpassungsfähigkeit von Viren möglich: Durch die Anpassung an seine Umwelt kann das Virus seine Lebensbedingungen aufrechterhalten und verändert damit sich und seine Umwelt.

Die soziale Relevanz für menschliche Gesellschaften erlangte SARS als zirkulierendes, heterogenes Akteur-Netzwerk von Viren und Zellen, Menschen und Tieren, Menschen und Technologien. Das Risiko der globalen Ausbreitung von SARS lässt sich also gesellschaftswissenschaftlich nicht allein durch Zirkulation symbolischer Medien der Kommunikation beschreiben, wie es der soziologische mainstream haben will. Es ist nicht nur die Kommunikation über SARS, sondern es ist SARS als handlungsmächtiges Akteur-Netzwerk, das gesellschaftliche Relevanz und gesellschaftlichen Wandel generiert. ${ }^{8}$ Damit werden aus reinen sozialen Tatsachen, die sich nur sozial erklären lassen, heterogene und individuelle kosmopolitische Ereignisse der Verknüpfung menschlicher und nicht-menschlicher, sozialer und nicht-sozialer Einheiten, die >das Soziale $<$ in Frage stellen und damit offen und begründungspflichtig erscheinen lassen. Akteure initiieren Netzwerke und Netzwerke Akteure: Akteure sind, d.h. sie ereignen sich als Akteur-Netzwerke.

Die Folgen solcher Netzwerke können hochgradig kontingent und widersprüchlich sein, wie man an der Virulenz der SARS-Netzwerke deutlich erkennen kann: So können Tier-Viren, die hochanschlussfähige Übersetzungsagenten darstellen, je nach Vermittlung für den menschlichen Organismus ungefährlich bleiben oder aber gefährliche Viren und kranke oder gar lebensbedrohte Körper produzieren und damit die Routinen menschlicher Organisation radikal in Frage stellen. Gleichzeitig wird dieses ursprüngliche SARS-Netzwerk in eine Vielzahl von - lokalen, regionalen, nationalen und transnationalen - sozio-technischen, biomedizinischen, wissenschaftlichen, massenmedialen Netzwerken etc. übersetzt.

Trotz ihres ereignislogischen, operationalen Charakters unterscheiden sich Akteur-Netzwerke deutlich von rein sozialen Ereignissen der Kommunikation (Luhmann 1986). Für sozialkonstruktivistische Beobachtertheorien, d.h. solche Ansätze, die die Zirkularität und Reflexivität von Beobachtungen von Beobachtungen ins Zentrum stellen, erscheint Sozialität als eine selbstreferentielle Operation in Differenz zum Nicht-Sozialen (Luhmann 1992; 1997). Hingegen rückt bei Akteur-Netzwerken Heterogenität, d.h. die Verknüpfung von Sozialem und Nicht-Sozialem ins Zentrum des Interesses. Dadurch wird gesellschaftlich wirksam, was beobachtungstheoretisch notwendigerweise im Dunkeln bleibt: Materialität, Natur, Menschen, Subjekte, Objekte, Körper und Sinne werden als heterogene Akteur-Netzwerke sichtbar.

Das Konzept >Akteur-Netzwerk< bricht mit der Durkheimschen Tradition, Soziales durch Soziales zu erklären, dadurch, dass gesellschaftliche Komplexität als emergierendes Phänomen zwar erhalten bleibt, die Kontingenz des Sozialen jedoch als Modus der Heterogenität sozialer Wirklichkeit und nicht als Modus der Differenz zu den nicht-sozialen Objekten anerkannt wird. Demnach ist >das Soziale< topologisch konzipiert - egal, ob es sich in menschlichen oder nicht-menschlichen Gesellschaften artikuliert - nicht ein »place, a thing, a domain, or a kind of stuff but a provisional movement of new associations (Latour 2005: 238). Unser methodisches Ziel ist dann auch nicht, sich von Subjekt und Objekt, von körperlichen Menschen und stofflichen Dingen gleichermaßen zu emanzipieren, wie das Beobachtertheorien tun. Sprechen wir von Subjekten und Objekten oder von Menschen und Dingen, dann als zeitlich-räumlich kontingente und heterogene Akteur-Netzwerke, also von Ereignissen, die das

8) Innerhalb der STS-Forschung wurde die Komplexität solcher heterogener Zusammenhänge bereits eindringlich beforscht (Callon 1998; Latour 1990; Law 1991; Law/Hassard 1999; Law/Mol 2002). 
Soziale in seiner Erklärungsbedürftigkeit und Fragwürdigkeit erst sichtbar machen. Als Akteur-Netzwerk wird SARS für menschliche Gesellschaften virulent, sobald es die Ordnungsmechanismen menschlichen Lebens und menschlicher Sozialität in Frage stellt und auch verändert. Akteur-Netzwerke erwirken die Bedingung der Möglichkeit von Kosmo-Politik. ${ }^{9}$

\section{SARS als kosmo-politisches Ereignis}

Die >heiße Phase < der Ausbreitung von SARS ist zunächst dadurch charakterisiert, dass weder die Erkrankung selbst noch deren Ursprung oder mögliche Evolution bekannt waren. So schreibt die kanadische Medscape Medical News:

»At the beginning of the outbreak, our biggest problem was our inability to recognize when a patient had SARS and our lack of understanding as to how SARS spread. This led to the spread of SARS throughout various hospitals. In particular, the transfer of unrecognized SARS patients between hospitals with resulting staff exposure was a major problem. (...) I think the problem initially was that it wasn't clear how far SARS had spread through the health care setting. (...) It was difficult to know at the time that the illness was not contained $\ldots \ll$ (Medscape Medical News, 13 April, 2003).

Dies hatte zur Folge, dass sich insbesondere Krankenhauspersonal - also Ärzte und Pflegekräfte - in großer Zahl infizierten und damit entscheidend an der Migration von SARS beteiligt waren. Es war zu Beginn des Ausbruchs die Unbekanntheit einer neuartigen Krankheit, die eine schnelle Ausbreitung von SARS entlang internationaler Flugrouten möglich machte. Damit verband sich dann auch das Risiko der pandemischen Ausbreitung einer zunächst atypischen und unbekannten, schweren Atemwegserkrankung.

Es kam nun darauf an, eine direkte Verbindung lokaler (interaktiver und organisatorischer), trans-lokaler (staatlicher) als auch transnationaler (WHO) Handlungszusammenhänge zu etablieren, um eine weitere Ausbreitung von SARS zu verhindern. Dies zog sehr unterschiedliche und teils hoch ambivalente soziale Effekte nach sich. In allen (!) betroffenen Ländern wurde in der Anfangsphase von SARS der desolate Zustand des öffentlichen Gesundheitssystems sichtbar: So gab es bspw. in nah beieinander liegenden Krankenhäusern eine Vielzahl von Infizierten, aufgrund von fehlenden Diagnoseverfahren, Erfassungssystemen und Meldestrukturen konnten jedoch keine Zusammenhänge zwischen diesen Fällen hergestellt werden. Auf diese Weise konnte sich die Krankheit dann in den Gesundheitseinrichtungen sehr schnell und unbemerkt ausbreiten. Krankenhäuser wurden zu hoch-virulenten Akteur-Netzwerken und es kam zur Schließung von Krankenhäusern, da sie eben eine zu große Gefahr für die öffentliche Gesundheit darstellten. Erst in der Zusammenarbeit mit der WHO konnten die entsprechenden Kontroll- und Meldesysteme installiert werden. Im Zuge der Bemühungen um die Eindämmung der Krankheit etablierte sich eine enge Zusammenarbeit mit den regionalen, nationalen und internationalen Gesundheitseinrichtungen. Dies erlaubte ein flächenübergreifendes Übersetzungsnetz von Informationen und so konnten sich dann auch lebenswichtige Schutzmaßnahmen (bspw. das Tragen von Mundschutz) wirksam durchsetzen und angewendet werden.

Wie entscheidend diese netzwerkartigen Formen der Zusammenarbeit für die Eindämmung einer zirkulierenden und virösen Krankheit waren, zeigt die Anfangsphase der Ausbreitung von SARS in China ganz deutlich. Die anfängliche Verweigerung der chinesischen Regierung einer Kooperation mit den internationalen Gesundheitsorganisationen führte da$\mathrm{zu}$, dass lebensrettende Maßnahmen ausblieben und die Zahl der Infizierten auf drastische

9) Akteur-Netzwerke sind immer dann nicht kosmo-politisch, wann immer sie gesellschaftliche Ordnungsmechanismen unhinterfragt lassen. 
Weise anstieg. Aufgrund fehlender Informationsstrukturen unterschätzte die chinesische Regierung sehr lange das Ausmaß der Ausbreitung der Krankheit. Erst der Druck der durch SARS verknüpften internationalen Solidargemeinschaft hat China zum Handeln bewegt.

Der Umgang mit dem Risiko einer globalen Ausbreitung von SARS zog dann auch sehr drastische Maßnahmen nach sich: dazu zählt bspw. eine radikale Einschränkung von Freiheitsrechten, indem Flugzeuge oder ganze Häuserblocks (z.B. die Wohnanlage >Amoy-Gardens $<$ in Hongkong) unter Quarantäne gestellt und Menschen in Turnhallen isoliert wurden. Schulen wurden geschlossen und öffentliche Plätze gesperrt. Die Verletzung solcher Anordnungen wurde in China gar mit der Androhung der Todesstrafe durchgesetzt.

Die WHO sprach erstmals in ihrer Geschichte eine Reisewarnung aus. Dies bedeutete nicht nur hohe wirtschaftliche Verluste für Reiseunternehmen und die Tourismus-Branche. Die Möglichkeit des schnellen Ortswechsels durch Züge und Flugzeuge barg in Verbindung mit SARS immer auch die Gefahr einer Ausbreitung der Krankheit in sich - auch über Ländergrenzen hinweg. Um die Ein- und Ausreise möglicher SARS-Infizierter kontrollieren zu können, kamen an Flughäfen aufwendige Screening-Verfahren zum Einsatz, um die Körpertemperatur ${ }^{10}$ der Passagiere zu registrieren. Entgegen der üblichen Routine an Flughäfen, wo der Besitz der nötigen Reisepapiere die Ein- und Ausreise regelt, war es also im Falle von SARS in erster Linie die Körpertemperatur, die darüber entschied, ob man frei-reisender citizen war und ein Flugzeug besteigen durfte oder nicht. Die Reisewarnung der WHO hatte zudem zur Folge, dass asiatische Unternehmen ihre ausländischen Mitarbeiter in ihre Heimatländer zurückschicken mussten. Hier stand die Gesundheit der Angestellten im Zentrum des Interesses für unternehmerische Entscheidungen.

Ebenso hat sich die wissenschaftliche Praxis im Umgang mit SARS verändert. Hier standen nicht länger die Exklusivität und die Erstveröffentlichung von Daten im Vordergrund. Es war im Gegenteil der rege Ressourcenaustausch und die enge Zusammenarbeit eines weltweiten Labornetzwerkes, die zur schnellen Diagnose des Krankheitserregers und damit letztendlich auch zur Eindämmung der Krankheit beitrugen, indem entsprechende Verhaltensregeln generiert wurden. Für die Medizin war für die Behandlung von Patienten die enge Zusammenarbeit mit der Forschung von entscheidender Bedeutung. Aufgrund der Neuartigkeit der Krankheit war mit gewohnten Methoden eine Diagnose nicht möglich. Erst durch Forschungsergebnisse, die den Krankheitserreger spezifizierten, konnten Diagnoseverfahren modifiziert und dann auch in eine wirksame Therapie umgesetzt werden.

Diese Beispiele im Umgang mit SARS machen deutlich, dass im Kontext der hohen, wenn auch unsicheren Erwartbarkeit und Wahrscheinlichkeit pandemischer Auswirkungen die gesellschaftliche, funktional differenzierte Übersetzungsleistung von Folgen auf systeminterne Entscheidungsprozesse an ihre Grenzen gerät. Das meint nicht, dass Mechanismen funktionaler Differenzierung verschwinden, sondern dass Problemdynamiken und Lösungsstrategien gesellschaftswirksam werden, die sich weder ohne noch allein mit Mitteln funktionaler Differenzierung beschreiben lassen. Vielmehr macht die Ausbreitung von SARS die topologische Komplexität gesellschaftlicher Problemlagen sichtbar, die zu translokalen, transregionalen und transnationalen Formen der wissenschaftlichen, politischen etc. Solidarität führte. Durch das Risiko einer globalen Ausbreitung von Ansteckungen und Erkrankungen mit möglicher Todesfolge wird ein Virus zu einem hoch-virulenten Akteur-Netzwerk, dessen konkrete Folgen die Grenzen der physiologischen und sozialen Organisation menschlicher Gesellschaften fragwürdig erscheinen lassen und die Restrukturierung tradierter sozialer Ordnungszusammenhänge initiieren.

10) Plötzliches ansteigendes und hohes Fieber gehört zu signifikanten Symptomen von SARS (Rickerts et al. 2003). 


\section{SARS und die Grenzen einer kosmopolitischen Soziologie}

Ausgehend von unserem methodologischen Anliegen, die topologische Komplexität von kosmo-politischen Ereignissen wie SARS zu benennen, haben wir SARS als Akteur-Netzwerk beschrieben: Netzwerke wie SARS benennen signifikante Akteure, die in der Entfaltung ihrer topologischen Komplexität zur Kosmo-politisierung gesellschaftlicher Wirklichkeitsbezüge und Ordnungskalküle beitragen. Dies entspricht zunächst auch der Motivation neuerer, kosmopolitisch ausgerichteter, soziologischer Perspektiven, die sich methodisch und theoretisch jenseits einer territorialen »Container-Perspektive« zu verorten versuchen (Beck 2003; 2004a; 2004b). Die Real-Logik dieses Ansatzes besteht nun darin, dass, im Unterschied zu einem normativen (philosophisch ausgelegten) Kosmopolitismus, ein empirisch-analytischer (sozialwissenschaftlich ausgelegter) kosmopolitischer Blick die real-existierenden globalen Dynamiken erfassen soll. Letzterer ist Ausdruck von institutionalisierter kosmopolitischer Reflexivität, d.h. einerseits der kritische Selbstbezug moderner Prozesse und andererseits das Bewusstwerden, die Erfahrung und Wahrnehmung individueller, sozialer und politischer Phänomene als globale Problemlagen und Zusammenhänge.

Aus einer kosmopolitischen Perspektive wäre SARS ein kosmopolitischer Akteur, sobald dessen Folgen global reflektiert werden und sie global lokale gesellschaftliche Auswirkungen haben. Im Sinne Becks sind die Folgen von Ereignissen wie SARS dann kosmopolitisch, sobald sie einen »institutionalisierten Kosmopolitismus« (Beck/Sznaider 2006) anzetteln, der die Lokalität globaler gesellschaftlicher Dynamiken und Problemlagen reflektiert und jenseits des Nationalstaates institutionell bearbeitet. SARS ist symptomatisch für eine »innere Kosmopolitisierung « (Beck 2002). Innere Kosmopolitisierung konstituiert sich aus der sozialen Logik der (Risiko-) Wahrnehmung der Folgen sozialer Praxis. Es ist der Umgang mit, die Wahrnehmung von und die Entscheidungen über SARS, die dessen Real-Logik bestimmen. Im Zuge innerer Kosmopolitisierung sind es also die Folgen der Wahrnehmung von SARS, die SARS so gefährlich erscheinen lassen. Somit weicht SARS als konstitutive Gefahr eines >Außen< der Immanenz >innerer<Globalität einer weltrisikogesellschaftlichen Wahrnehmungsdynamik, die kosmopolitisierte Praktiken auslöst (Beck 2002; 2005). Das haben die antizipierten Folgen von SARS, wie die oben angeführten Beispiele zeigen, in der Tat bewirkt: Es ist das Risiko, d.h. die Virtualität einer globalen SARS-Epidemie (und nicht eine tatsächliche Pandemie!), welche Wissenschaft, Wirtschaft oder Politik in ihren lokalen, regionalen und nationalen Routinen irritiert und in Frage stellt. In Bezug auf die Gefahr einer weltweiten Ausbreitung von SARS werden die jeweiligen bestehenden Strategien in der Kombination und Vernetzung vielfältiger Praktiken neu konstituiert.

Dennoch unterscheidet sich unser Verständnis von SARS als kosmo-politischem Ereignis deutlich von den normativen Prämissen kosmopolitischer Soziologie im Sinne Becks. Letzterer versteht Kosmopolitik als strukturellen Zwang selbsterzeugter (industriegesellschaftlicher) Folgen, die dann eine >cosmopolitan condition< (Beck/Sznaider 2006) heraufbeschwören und die Bedeutung der »universellen Norm menschlicher Gleichheit « (Beck/Grande 2004: 29) durchsetzen. Man ist nicht von Natur aus Kosmopolit, sondern man wird strukturell zum Kosmopoliten gemacht. Der Kosmopolit, so Beck, ist dann »sowohl Bürger des Kosmos - Weltbürger - als auch Bürger der Polis - Stadt- und Staatsbürger « (ebd.). Der »kosmopolitische Blick « initiiert daraus »einen» Kosmos (>Welt $<)$, der durch den »orts-polygamen « Anderen (Beck 2004a) - den Welt- und Staat/Stadt-Bürger - politisiert und pluralisiert wird. Der darauf justierte kosmopolitische Blick des Sozialwissenschaftlers dient dabei als zentrales, öffentliches Sprachrohr des Kosmopolitischen.

Es ist gerade die Dialektik von Struktur und Handlung als Ausdruck von Kosmopolitisierung, welche methodologisch immer noch im Sinne klassischer globaler Soziologie gedacht ist, indem diese 
(1) die Folgen von sozialer Praxis immer nur sozial erklären kann, und darüber hinaus

(2) die kosmopolitische Topologie einer öffentlichkeitsgenerierenden

Folgenlogik in die Anerkennung der sozial erwirkten universellen Gleichheit (der Andersheit) des Menschen fließen lässt.

Die so heraufbeschworene Dialektik zwischen Universalismus und Partikularismus der Beckschen inneren Kosmopolitisierung unterliegt einem humanistisch geprägten Stil, der gerade die topologische Komplexität von Akteur-Netzwerken - als Verknüpfung von menschlichen und nicht-menschlichen wie auch sozialen und nicht-sozialen Einheiten - nicht in den Blick bekommt. Der kosmopolitische Andere ist der gute oder schlechte menschliche Bürger, der durch die globalen Folgen seines eigenen Tuns strukturell gezwungen wird, die Grenzen seiner sozialen Handlungen neu zu überdenken und zu verändern. Dass AkteurNetzwerke Menschen sind, ist in unserer Argumentation kosmo-politischer Ereignisse hingegen nur eine mögliche Artikulation topologischer Komplexität unter vielen.

SARS nun - so unsere These - stellt als kosmo-politisches Ereignis gerade die Idee von einer >inneren Kosmopolitisierung < in Frage, indem es zweierlei erkennen lässt:

(1) Die Folgen sozialer Praxis lassen sich nicht mehr allein sozial erklären, sondern stellen das >Soziale< und ihre methodologische Begrenzung durch die klassische sozialwissenschaftliche Beschreibung in Frage.

(2) Die Öffentlichkeitswirksamkeit von SARS ist nicht allein den Folgen der sozialen Risikowahrnehmung geschuldet, sondern verdankt sich der Handlungsmacht von Akteur-Netzwerken. Als solches wird SARS zu einem gesellschaftlich hochrelevanten Ereignis.

Strukturell erzwungener Kosmopolitismus ermöglicht das Vertrauen in die menschliche Andersheit des Anderen als politischen Akteur. Durch Kosmopolitisierung sitzen wir alle wie unterschiedlich wir auch sein mögen - im gleichen Boot. Mit dem Ergebnis, dass sich die universelle Gleichheit der >Menschen « nicht nur der Kosmopolitisierung auf unerklärte Weise entzieht, sondern sie auch in ihrer empirischen, politischen Rolle als >Subjekte< oder $>$ Kollektiveく überschätzt. Demgegenüber werden nicht-menschliche Akteure, wie z.B. SARS, in ihrer politischen Handlungsmacht und Bedeutung deutlich unterschätzt. Hier scheint uns doch eher ein abstrakt bleibender, normativ-philosophischer und damit a-historischer, humanistischer Kosmopolitismus am Werk, als die Real-Logik einer empirisch-analytisch ausgelegten kosmopolitischen Sozialwissenschaft. Vielmehr komplettiert Becks Kosmopolitismus die Normativität des Kantschen subjektiven Kosmopolitismus, indem er diesen soziologisiert, d.h. als strukturell erzwungen beschreibt.

Das zentrale Moment der Beckschen Argumentation für die Wende hin zu einem >methodologischen Kosmopolitismus> besteht darin, dass im Zuge der Folgen gesellschaftlicher Modernisierung die Basis-Institutionen und Basis-Prämissen in Frage gestellt werden (Beck/ Sznaider 2006). Verstehen wir Becks Anliegen richtig, dann zwingt dies methodologisch zwar den Blick global, also jenseits des Containers des Nationalstaates auszurichten. Dies lässt aber das methodologische Basisprinzip klassischer Soziologie unhinterfragt, >das Soziale< durch die Unterscheidung vom >Nicht-Sozialen< zu bestimmen. Kosmopolitik beginnt in der Tat, wo die Unterscheidung zur Natur hinfällig wird (Beck 1986, 1993). Dies schließt aber die methodologisch erwirkte Natur des >Sozialen< und seiner menschlichen Akteure mit ein. Kosmo-politische Ereignisse stellen nicht nur die Methodologie des Sozialen klassischer Soziologie in Frage, sondern auch das Basisprinzip der Dialektik zwischen Handlung und Struktur eines >methodologischen Kosmopolitismus< und dessen Universalisierung der Andersheit menschlicher Gleichheit. 
Im Sinne Bruno Latours (1995; 2005) verweist der Becksche Kosmopolitismus auf eine typisch moderne Strategie, >Mensch < wie >Gesellschaft< als rein soziale Kollektive auszuweisen, die den Umgang mit, die Bedeutung von und den Unterschied zu nicht-menschlichen Wesen und zur Natur bestimmen. Becks Dialektik spricht zwar von einer selbsthergestellten realen Struktur und >objektiven Gefahrenlage<, welche die kosmopolitische Dimension global ins Bewusstsein bringt. Aber ganz im Duktus klassischer soziologischer Methodologie ist es an der Gesellschaft (im Gegensatz zur Natur) - sei es das menschliche Individuum oder Kollektive (Organisationen, Institutionen) - dies zu erkennen und zu verändern. Dabei vergisst Beck die Übersetzungsleistungen zwischen Sozialem und Nicht-Sozialem, zwischen Natur und Kultur kosmo-politischer Ereignisse; d.h. die ständige Produktion von >Mischwesen<, >Hybriden< oder eben >Akteur-Netzwerken<, welche die hoch-komplexen Dynamiken der Vermittlung sozialer und nicht-sozialer Zusammenhänge, d.h. die Fabrikation von Natur/ Kulturen, aktualisieren.

Die Wissenschaften sind für Latour das Paradebeispiel dafür, beides zu leisten: Natur und Kultur zu mischen und diese daraufhin zu trennen, d.h. Natur vs. Kultur und Natur/Kultur zu produzieren (Latour 1987; 1988; Stengers 1997). Auch im Falle der Identifizierung von SARS waren die Praxen der Wissenschaften maßgeblich beteiligt, >Natur zirkulieren zu lassen und damit in die Welt zu bringen, d.h. sozial anschlussfähig zu übersetzen, mit dem Ziel, die Ausbreitung des Virus einzudämmen. Als kosmo-politisches Ereignis hat SARS dazu beigetragen, wissenschaftliche Praxis und Routinen zu hinterfragen und jenseits lokaler und nationaler Grenzen in offene Vernetzungsstrategien einzubinden. Jenseits der Dialektik von Handlung und Struktur werden dadurch Akteur-Netzwerke sichtbar, die menschliche und nicht-menschliche, soziale und nicht-soziale Einheiten verknüpfen und global zirkulieren lassen. Ein >methodologischer Kosmopolitismus< trägt so eher zum Unsichtbarmachen von sozial/nicht-sozialen Hybriden bei, gerade weil er die Normativität moderner Strategien den Folgen der Globalisierung aussetzt.

Das Charakteristikum einer modernen Perspektive besteht darin, dass man sich für die eine (Gesellschaft) oder die andere Seite (Natur) der Trennung entscheiden kann. Sie benennt das soziologisch gut verstandene Grundphänomen der Kontingenz erstmoderner Zusammenhänge (Luhmann 1992; Nassehi 2003; Makropolus 2004). Dennoch bedeutet die Entscheidung für die eine Seite (Gesellschaft) die Anerkennung von Modernität und die Entscheidung für die andere Seite (Natur) die Nicht-Anerkennung von Modernität. Das Wissen darüber, dass man sich für beide Seiten entscheiden kann, und die eine Seite dort beginnt, wo sie sich von der anderen unterscheidet, wird als Basis-Prinzip der Trennung moderner von nicht-moderner Praxis zu Grunde gelegt. Man kann gar nicht mehr anders als modern zu sein, auch dann nicht, wenn der Mensch oder die Gesellschaft(en) sich gegen die Moderne entscheiden und sich und die Welt von Gott geschaffen oder von der Natur bestimmt erklären, da man weiß, dass es der gesellschaftliche Mensch ist, der darüber entschieden hat. Damit begründet sich seit Berkeley und Kant der moderne Mensch als Beobachter seiner Natur und im soziologischen Diskurs >das Soziale< als dessen gesellschaftliches Pendant. Versucht man jedoch beide Dynamiken (Trennung, Vermittlung) als eben ein Akteur-Netzwerk zusammen zu denken, also Symmetrie zwischen Natur und Kultur zu gewähren, hört man auf, exklusiv modern zu sein. Man beschreibt Natur/Kulturen, führt Heterogenität ein und markiert den Übergang in topologische Komplexität, die die Fabrikation von nicht-modernen Mischwesen, von mediativen Akteur-Netzwerken wie SARS in gleicher Weise für die gesellschaftliche Praxis und ihre Beobachtungsstrategien bedenkt, wie dies die Komplexität moderner Kosmopolitik- und Kontingenzformeln tut. 


\section{Kosmo-politische Topologie}

In diesem Schlusskapitel wollen wir uns mit der topologischen Dimension des Begriffs der Kosmo-Politik als Ereignis befassen. Am Beispiel der Migration von SARS haben wir versucht, dessen topologische Bedeutung zu beschreiben. Der Zirkulation von SARS gelang es, eine Vielzahl von Akteur-Netzwerken sichtbar zu machen, die >Lokalität< und >Globalität< als Folge lokaler Praxis ins Zentrum des Interesses stellen. Akteur-Netzwerke benennen einen praktizierten Raum, der auf Versammlungen, auf Zusammenkünfte hinweist. Topologisch gehaltvoll ist nicht der Raum, in dem etwas stattfindet, sondern umgekehrt, die Versammlungen und Zusammenkünfte bestimmen die Besonderheiten, die Komplexität solcher Akteur-Netzwerke. Somit wird durch Akteur-Netzwerke sichtbar, dass das Lokale nicht lokal und das Globale nicht global ist. Das so verstandene Ereignen von Lokalität und Globalität als kontingente und heterogene Praxis benennt die Dis/Lokalisierung der Unterscheidung lokal vs. global. Damit wird sowohl der Kosmos des Lokalen als auch der des Globalen in Frage gestellt.

Hannah Arendt $(1960 ; 1993)$ hat die Praxis solcher Dis/Lokalisierungen als immanent politischen, als öffentlichen Raum beschrieben. Dis/Lokalisierung meint bei Arendt, dass Menschen sich versammeln und sich dadurch ihre individuelle, prä-politische Vielfalt und Andersheit durch gemeinsame Praxis als politische Macht (Arendt 1993) aktualisiert. Bei Arendt sind sowohl das Politische als auch Machtverhältnisse Errungenschaften von menschlicher Praxis, die den Menschen von seiner natürlichen Individualität in einen handelnd-öffentlichen und somit politischen Raum transformiert. Damit wird der Mensch vom individuellen prädisponierten Wesen zum Gemeinschaftswesen, das Öffentlichkeiten erzeugt und dadurch gesellschaftliche Gestaltungsspielräume und Veränderungspotentiale, also im Arendtschen Sinne politische Freiräume durch Handeln aktualisiert. Der Arendtsche Politikbegriff ist bereits topologisch gedacht, da er sich nicht auf einen (euklidisch) abgegrenzten politischen Raum bezieht. Er umreißt vielmehr einen humanistisch konzipierten Kosmopolitismus, der immanent ereignislogisch angelegt ist, d.h. politischen Freiraum als menschliches Handeln, als Werden, als Ereignen von pluralen Öffentlichkeiten initiiert.

Damit ist bei Arendt Handeln an die »Grundbedingung der Natalität enger gebunden als Arbeiten und Herstellen. « Arbeiten und Herstellen komplettieren die vita activa der human condition und bestimmen die »menschlichen Grundtätigkeiten « mit ihren korrespondierenden »Grundbedingungen « und machen damit die »Bedingtheit des Menschen« aus. Während die Tätigkeit der Arbeit die Grundbedingungen des Lebens bestimmt, entspricht Herstellen der Weltlichkeit, d.h. der »Angewiesenheit menschlicher Existenz auf Gegenständlichkeit und Objektivität« (Arendt 1960: 14). Demgegenüber meint Handeln und ihre Grundbedingung der Pluralität des Menschen die Tätigkeit, welche sich »ohne die Vermittlung von Materie, Materialen und Dingen direkt zwischen Menschen abspielt« (ebd., Hervorhebung S/P). Bei Arendt umschreibt Handeln »die politische Tätigkeit par excellence«, so Arendt, und »bedarf einer Pluralität, in der zwar alle dasselbe sind, nämlich Menschen, aber dies auf die merkwürdige Art und Weise, dass keiner dieser Menschen je einem anderen gleicht, der einmal gelebt hat oder lebt und leben wird (ebd.: 15f).

Arendt hat deutlich erkannt, dass »(j)ede menschliche Tätigkeit (...) in einer Umgebung von Dingen und Menschen « (Arendt 1960: 26) sich lokalisiert, sieht jedoch in der Koexistenz von Menschen alleine das machtvolle Ereignis des Politischen und damit die Möglichkeit, neu und anders anfangen zu können. Handeln im Arendtschen Sinne anerkennt zwar die terra incognita unserer Tätigkeiten, d.h. das enge Verhältnis von biologischem Leben und technischer Konstruktion(en) mit menschlichem Handeln. Die kosmo-politische Dimension der Arendtschen vita activa beschränkt sich aber auf das menschliche Handeln, das alleinig 
imstande ist, bestehende Kosmologien, d.h. tradierte unhinterfragte soziale Ordnungszusammenhänge zu politisieren und neu zu gestalten.

Interessanterweise ist der Arendtsche Handlungsbegriff normativ im Unterschied zur Sozialität konzipiert und so aus der Sicht sozialwissenschaftlicher Methodologie ganz und gar nicht-modern konzipiert, da er politische Handlung außerhalb des Sozialen lokalisiert. Sozialität im Arendtschen Sinne meint die prä-politische Pluralität der Menschen. Sozialität ermöglicht zuallererst die Macht des politischen Diskurses, des sprachlichen Disputs, der sich aber dadurch in situ von Sozialität unterscheidet. Handeln politisiert Sozialität, es stellt Sozialität in Frage, anstatt in ihr aufzugehen, wie es die sozialwissenschaftliche Methodologie will. Durch politische Praxis wird Sozialität dis/lokalisiert, es wird zum Ereignis. Das Soziale als politisiertes Ereignis »erleuchtet seine eigene Vergangenheit, aber es kann niemals aus ihr abgeleitet werden« (Arendt in Kallscheuer 1993: 153).

In unserer bisherigen Diskussion hatten wir argumentiert, dass die methodologische Basisunterscheidung >sozial/nicht-sozial< sozialwissenschaftlicher Selbstbeschreibungen durch SARS fragwürdig wird und es sukzessive erlaubt, die topologische Komplexität in der Verknüpfung sozialer und nicht-sozialer Zusammenhänge zu sehen. Dadurch wird das Ende des Sozialen als Einheit der Differenz sozial/nicht-sozial virulent und ermöglicht, die kontingenten und heterogenen Möglichkeitsräume gesellschaftlichen Wandels als multiples AkteurNetzwerk-Ereignis zu beschreiben. Der Arendtsche kosmo-politische Handlungsbegriff kann dies ebenso leisten, erlaubt es jedoch nicht, den politischen Raum als komplexe Vermittlung von menschlichen und nicht-menschlichen Akteuren zu denken. Vielmehr wird ja gerade bei Arendt >Handeln< als rein menschliche (d.h. sprachliche) Tätigkeit beschrieben.

Unsere Diskussion jedoch zeigt, dass SARS die Basisunterscheidungen der Arendtschen vita activa ebenfalls korrumpiert. Im Zuge der Zirkulation von SARS politisiert sich Begriff und Praxis des Politischen selbst. Als Bedingungen des Kosmo-Politischen ist nun nicht mehr die Kantisch/Arendtsche/Becksche Pluralität des Menschen, sondern die Heterogenität und Multiplizität menschlich/nicht-menschlicher Vermittler getreten. SARS ist hierfür nur ein Beispiel, um auf die Heterogenität öffentlichkeits-generierender und öffentlichkeitswirksamer Praxis kosmo-politischer Ereignisse zu verweisen, die jenseits der Dialektik von Handlung und Struktur die topologische Komplexität von Natur/Kulturen entfalten.

\section{Literatur}

Arendt, H. (1960): Viva actica oder Vom tätigen Leben, Stuttgart.

Arendt, H. (1993): Macht und Gewalt, München.

Baehr, P. (2005): Social extremity, communities of fate, and the sociology of SARS, in: European Journal of Sociology 46, S. 179-211.

Barabasi, A.L. (2002): Linked: the new science of networks, New York.

Bateson, G. / Bateson, M.C. (1993): Wo Engel zögern. Unterwegs zu einer Epistemologie des Heiligen, Frankfurt am Main.

Beck, U. (1986): Die Risikogesellschaft. Auf dem Weg in eine andere Moderne, Frankfurt am Main.

Beck, U. (1993): Die Erfindung des Politischen. Zu einer Theorie reflexiver Modernisierung, Frankfurt am Main.

Beck, U. (2002): Macht und Gegenmacht im globalen Zeitalter, Frankfurt am Main.

Beck, U. (2003): Toward a new critical theory with a cosmopolitan intent, in: Constellations 10, S. 435468.

Beck, U. (2004a): Der kosmopolitische Blick oder: Krieg ist Frieden, Frankfurt am Main. 
Beck, U. (2004b): Cosmopolitical realism: on the distinction between cosmopolitanism in philosophy and the social sciences, in: Global Networks 4, S. 131-156.

Beck, U. (2005): World risk society and the foundations of transnational politics, in: E. Grande / L.W. Pauly (Hrsg.): Complex sovereignty, Toronto, S. 22-47.

Beck, U. / Grande, E. (2004): Das kosmopolitische Europa, Frankfurt am Main.

Beck, U. / Sznaider, N. (2006): Unpacking cosmopolitanism for the social sciences: a research agenda, in: British Journal of Sociology 57, S. 1-23.

Berg, M. / Mol, A. (Hrsg.) (1998): Differences in medicine. Unravelling practices, techniques, and bodies, Durham, London.

Breiman, R. F. et. al (2003): The role of China in the quest to define and control Severe Acute Respiratory Syndrome, in: Emerging Infectious Diseases 9, S. 1037-1041.

Callon, M. (1991): Techno-economic networks and irreversibility, in: J. Law (Hrsg.): A sociology of monsters. Essays on power, technology and domination, London, S. 132-161.

Callon, M. (Hrsg.) (1998): Laws of markets, Oxford.

Callon, M./ Law, J. (2004): Introduction: absence - presence, circulation, and encountering in complex space, in: Environment and Planning D: Society and Space 22, S. 3-11.

Cambell, A. (2004): The SARS commission first interim report. SARS and public health legislation. Ontario.

Cussins, C. (1998): Ontological choreography: agency for women patients in an infertility clinic, in: M. Berg/ A. Mol (Hrsg.): Differences in medicine. Unravelling practices, techniques and bodies, Durham, London, S. 166-201.

Czarniawska, B. / Sevón, G. (Hrsg.) (2005): Global ideas: how ideas, objects and practices travel in the global economy, Liber, Malmö.

Doerr, H.W. (2003): SARS - eine neue Infektionskrankheit (gehalten als öffentlicher Vortrag am 27. Juni 2003), in: Jahrbuch der Akademie der Wissenschaften zu Göttingen 2003, Göttingen.

Durkheim, É. (1961): Regeln der soziologischen Methode, Neuwied.

Gleick, J. (1999) Faster. The acceleration of just about everything, München.

Hetherington, K. / Lee, N.M. (2000): Social order and the blank figure, in: Environment and Planning D: Society and Space, 18, S. 169-184.

Jasanoff, S. / Long Martello, M. (Hrsg.) (2004): Earthly politics: local and global in environmental governance, Cambridge.

Kallscheuer, O. (1993): Der verweigerte Dialog. Hannah Arendt und die europäischen Intellektuellen, in: P. Kemper (Hrsg.): Die Zukunft des Politischen. Ausblicke auf Hannah Arendt, Frankfurt am Main, S. 142-179.

Kant, I. (1997): Schriftenreihe zur Anthropologie, Geschichtsphilosophie, Politik und Pädagogik, Werkausgabe Band 12, Frankfurt am Main.

Latour, B. (1987): Science in action. How to follow scientists and engineers through society, Cambridge.

Latour, B. (1988): The pasteurization of France, Cambridge.

Latour, B. (1990): Drawing things together, in: M. Lynch / S. Woolgar (Hrsg.): Representation of scientific practice, Cambridge, S. 107-124.

Latour, B. (1995): Wir sind nie modern gewesen, Berlin.

Latour, B. (1996): On interobjectivity, in: Mind, Culture and Activity 3, S. 228-245.

Latour, B. (1999): On recalling ANT, in: J. Law/J. Hassard (Hrsg.): Actor Network Theory and after, Oxford, S. 15-25.

Latour, B. (2000): When things strike back: a possible contribution of science studies to the social sciences, in: British Journal of Sociology 51, S. 107-124.

Latour, B. (2001): Gabriel Tarde und das Ende des Sozialen, in: Soziale Welt 52, S. 361-376.

Latour, B. (2005): Reassembling the social: an introduction to actor-network-theory, Oxford. 
Law, J. (Hrsg.) (1991): A sociology of monsters. Essays on power, technology and domination, London.

Law, J. (1999): After ANT: complexity, naming and topology, in: J. Law / J. Hassard (Hrsg.): Actor network theory and after, Oxford. S. 1-14.

Law, J. (2002a): Aircraft stories. Decentering the object in technoscience, Durham.

Law, J. (2002b): And if the global were small and non- coherent? Method, complexity and the baroque, Environment and Planning D: Society and Space 21, S. 13-26.

Law, J. (2004): After method: mess in social science research, London.

Law, J. / Hassard, J. (Hrsg.) (1999): Actor network theory and after, Oxford.

Law, J. / Mol, A. (2001): Situating technoscience: an inquiry into spatialities, in: Environment and Planning D: Society and Space 19, S. 609-621.

Law, J. / Mol, A. (Hrsg.) (2002): Complexities: social studies of knowledge practices, Durham.

Lee, N. / Brown, S. (1994): Otherness and the actor network: the undiscovered continent, in: American Behavioural Scientist 36, S. 772-790.

van Loon, J. (2002): Risk and technological culture. Towards a sociology of virulence, London, New York.

Luhmann, N. (1986): Soziale Systeme. Grundriß einer allgemeinen Theorie, Frankfurt am Main.

Luhmann, N. (1992): Beobachtungen der Moderne, Opladen.

Luhmann, N. (1997): Die Gesellschaft der Gesellschaft. 2 Bände, Frankfurt am Main.

Medscape Medical News, 13 April, 2003, verfügbar über: http://www.medscape.com

Mol, A. (2002): The body multiple. Ontology in medical practice, Durham.

Makropoulos, M. (2004): Kontingenz Aspekte einer theoretischen Semantik der Moderne, in: European Journal of Sociology 45, S. 369-399.

Moreira, T. (2004a): Surgical nomads: a social topology of the operating room, in: Environment and Planning D: Society and Space 22, S. 53-69.

Moreira, T. (2004b): Coordination and embodiment in the operating room, in: Body and Society 10, S. 109-129.

Nassehi, A. (2003): Geschlossenheit und Offenheit, Frankfurt am Main.

Ong, A. (2004): Assembling around SARS: technology, body heat, and political fever in risk society, in: A. Poferl/ N. Sznaider (Hrsg.): Ulrich Becks kosmopolitisches Projekt. Auf dem Weg in eine andere Soziologie, Baden-Baden, S. 81-89.

Ong, A. / Collier, S.J. (Hrsg.) (2004): Global assemblages: technology, politics, and ethics as anthropological problems, Oxford.

Petryna, A. (2002): Life exposed: biological citizens after Chernobyl, Princeton.

Preda, A. (2004): Aids, rhetoric, and medical knowledge, Cambridge.

Preiser, W. (2004): SARS - Ein Virus hält die Welt in Atem, in: Brockhaus Enzyklopädie Jahrbuch 2003, Leipzig, Mannheim, S. 288-291.

Preiser, W. / Rabenau, H.F. / Doerr, H.W. (2002): Viren, Viruserkrankungen. Synopsis der Epidemiologie, Klinik, Diagnostik und Therapie viraler Erkrankungen, Steinen.

Rickerts, V. et al. (2003): Klinik und Behandlung des schweren akuten respiratorischen Syndroms, in: Deutsche Medizinische Wochenschrift 128, S. 1109-1114.

Schillmeier, M. (im Erscheinen): Dis/abling spaces of calculation - Blindness and money in everyday life, in: Environment and Planning D: Society and Space.

Serres, M. (2005): Atlas, Berlin.

Simmel, G. (1989): Philosophie des Geldes. Gesamtausgabe Band 6, Frankfurt am Main.

Stengers, I. (1997): Die Erfindung der modernen Wissenschaften, Frankfurt am Main, New York.

Stengers, I. (2004) The cosmopolitical proposal. mimeo. Université Libre de Bruxelles.

Strathern, M. (1991): Partial connections, Savage. 
Strathern, M. (1992): After nature, Cambridge.

Taylor, M.C. (2001): The moment of complexity: engineering network culture, Chicago.

Thrift, N. (1996): Spatial formations, London.

Turner, B.S. (2006): Classical sociology and cosmopolitanism: a critical defence of the social, in: British Journal of Sociology 57, S. 133-151.

Urry, J. (2000): Sociology beyond societies. Mobilities for the twenty-first century, New York.

Urry, J. (2002): Mobility and proximity, in: Sociology 36, S. 255-274.

Urry, J. (2003): Global complexity, London.

Urry, J. (2005): The complexities of the global, in: Theory, Culture and Society 22, S. 235-254.

Whitehead, A.N. (1967): Science and the modern world, New York.

WHO (2003 a): World Health Organization. Update 95 - SARS: Chronology of a serial killer, verfügbar über: http://www.who.int/csr/don/2003_07_04/en/.

WHO (2003 b): Epidemic and Pandemic Alert and Response, SARS - multi-country outbreak - Update 27, 11 April 2003, S. 9, verfügbar über: http://who.int/csr/en/.

Michael Schillmeier, Ph.D. e-mail: m.schillmeier@lmu.de

Dipl.-Soz. Wiebke Pohler

Wiebke.Pohler@soziologie.uni-muenchen.de

Ludwig-Maximilians-Universität München Institut für Soziologie

Konradstraße 6

80801 München 